\title{
Advantages of bariatric medicine for individualized prevention and treatments: multidisciplinary approach in body culture and prevention of obesity and diabetes
}

\author{
Dimiter V. Dimitrov • Valkan Ivanov • Maria Atanasova
}

Received: 13 May 2011 / Accepted: 27 June 2011 / Published online: 14 July 2011

(C) European Association for Predictive, Preventive and Personalised Medicine 2011

\begin{abstract}
Bariatric surgery is a component of the multimodal treatment of obesity, which consists of multidisciplinary evaluation and diagnosis, conservative and surgical treatments, and lifelong follow-up care. The current guideline extends the BMI-based spectrum of indications that was previously proposed (BMI greater than $40 \mathrm{~kg} / \mathrm{m}(2)$, or greater than $35 \mathrm{~kg} /$ $\mathrm{m}(2)$ with secondary diseases) by eliminating age limits, as well as most of the contraindications. A prerequisite for surgery is that a structured, conservative weight-loss program has failed or is considered to be futile. Type 2 diabetes is now considered an independent indication under clinical study conditions for patients whose BMI is less than $35 \mathrm{~kg} / \mathrm{m}(2)$ (metabolic surgery). The standard laparoscopic techniques are gastric banding, gastric bypass, sleeve gastrectomy, and biliopancreatic diversion. The choice of procedures is based on knowledge of the results, long-term effects, complications, and individual circumstances. Structured lifelong follow-up should be provided and should, in particular, prevent metabolic deficiencies.
\end{abstract}

Keywords Obesity · Bariatric medicine $\cdot$ Individualized treatment Predictive diagnostics $\cdot$ Preventive measures . Cost-effective personalized medicine

\section{Introduction}

Obesity and its associated diseases are epidemic that represent a major threat to human health. In the last two decades an explosive increase in the number of people diagnosed with

D.V. Dimitrov is a National Representative of EPMA in Bulgaria.

D. V. Dimitrov $(\bowtie) \cdot$ V. Ivanov $\cdot$ M. Atanasova

Nutrigenomics Center Varna, Medical University,

Varna, Bulgaria

e-mail: dimiter.v.dimitrov@gmail.com diabetes has been observed worldwide and the global figure of affected individuals is expected to rise from currently 150 million to 300 million in 2025 [1]. The rapidly escalating number of affected patients at even very young age poses a tremendous burden on the public health system and a substantial reinforcement of research activities including transnational cooperation between scientists from different disciplines is urgently required to avoid a socio-economic disaster. For patients with obesity and type 2 diabetes, bariatric surgery is by far the most effective treatment-it is, indeed, the only form of treatment that can put patients into full long-term remission [2]. In this article we will review and highlight the importance of bariatric surgery as a way to cut diabetes costs at present. Finally, we will discuss public health implications. Until a successful non-surgical means for preventing and reversing obesity is developed, bariatric surgery appears to be the only intervention that can result in a sustained reversal of both obesity and type 2 diabetes mellitus in most patients receiving it. One of the key recommendations is the need for a multidisciplinary bariatric team to oversee the ongoing needs of patients after surgery, as well as to build a weight maintenance program.

The term bariatric medicine refers to gastrointestinal surgical interventions and subsequent multidisciplinary lifelong followup conducted to produce sustainable weight loss. Bariatric surgery is a term derived from the Greek words: weight and treatment. Currently, most obesity clinics and bariatric centers favor the Lap Band adjustable gastric banding procedure and the proximal Roux-en-Y Gastric Bypass (Table 1).

\section{Pre-operative patient selection and preparation}

The generally accepted indication for gastrointestinal surgery for weight loss is BMI (body mass index) $>40$ or 
Table 1 Description of bariatric surgery procedures

\begin{tabular}{|c|c|}
\hline Bariatric technique & Description of the procedure \\
\hline $\begin{array}{l}\text { Adjustable Gastric Binding } \\
\text { (AGB) }\end{array}$ & $\begin{array}{l}\text { AGB involves creating of a small upper gastric pouch }(15-45 \mathrm{ml} \text { in volume }) \text {, by placing a band around the upper } \\
\text { stomach. The primary aim is to create a mechanical restriction that limits the passage of the food. This enables } \\
\text { the patients to limit portion sizes without feeling undue hunger. Very recently wireless, telemetric adjustable } \\
\text { system has been developed for periodic adjustments of the band. }\end{array}$ \\
\hline $\begin{array}{l}\text { Roux-en-Y Gastric Bypass } \\
\text { (RYGB) }\end{array}$ & $\begin{array}{l}\text { In a RYGB, the cardia is separated from the remainder of the stomach, creating a small gastric reservoir measuring } \\
\text { approximately } 10 \mathrm{ml} \text {. This reservoir is then anastomosed to a segment of the proximal jeunum. The small gastric } \\
\text { reservoir restricts food intake and causes degree of malabsoption. }\end{array}$ \\
\hline $\begin{array}{l}\text { Bileopancreatic Diversion } \\
\text { (BPD) }\end{array}$ & $\begin{array}{l}\text { In a BDP, a subtotal gastrectomy is performed, leaving gastric pouch } 200-500 \mathrm{ml} \text { in volume. The distal segment } \\
\text { of the small intestine is anastomosed to the gastric remnant and the proximal segment is anastomosed to the } \\
\text { distal ileum } 50 \mathrm{~cm} \text { from the ileocecal valve. }\end{array}$ \\
\hline
\end{tabular}

BMI > 35 complicated by weight-related co-morbidities [3]. The clinician should view these parameters as a guide rather then a rule, as each patient should be assessed on individual basis (Fig. 1). Before patients undergo bariatric surgery, it is very important to address their expectations. Pre-operative and postoperative education must aim to confront the common patient belief that post-surgical weight loss will be effortless and that they will achieve their 'dream' weight. Patients need to understand that the primary goal is to reduce the risks of morbidity and mortality associated with their pre-surgical weight. Patients can be asked to describe their weight, ideal weight and height; actual weight and height can be assessed using a standard medical scale. After obtaining the surveyed information, the patient can provide motivational feedback by addressing three topics of diet and exercise-related health risks: heart disease (specifically myocardial infarction), hypertension (and specifically risk for stroke), and diabetes. Next, patients can elicit questions and provide short-term realizable goals with respect to diet and exercise habits. Following the intervention, participants can be asked whether they would participate in a longitudinal study of brief motivational intervention. Each patient engages understanding of the intervention on a scale from 1 to 10 , with 1 being the least engaged and 10 being the most engaged. Patients should understand that undergoing baritaric surgery is very significant commitment that also requires dramatic lifestyle changes. In addition, they should know that for a severely obese person with clinically significant end-organ damage, the risk of death without surgery within 1 year is about 10 times that of dying of the surgery itself.

\section{Biomarkers for predictive diagnostics and preventive measures}

Bariatric surgery is increasingly used as a strategy to reduce bodyweight and thereby ameliorate risk factors for cardio-

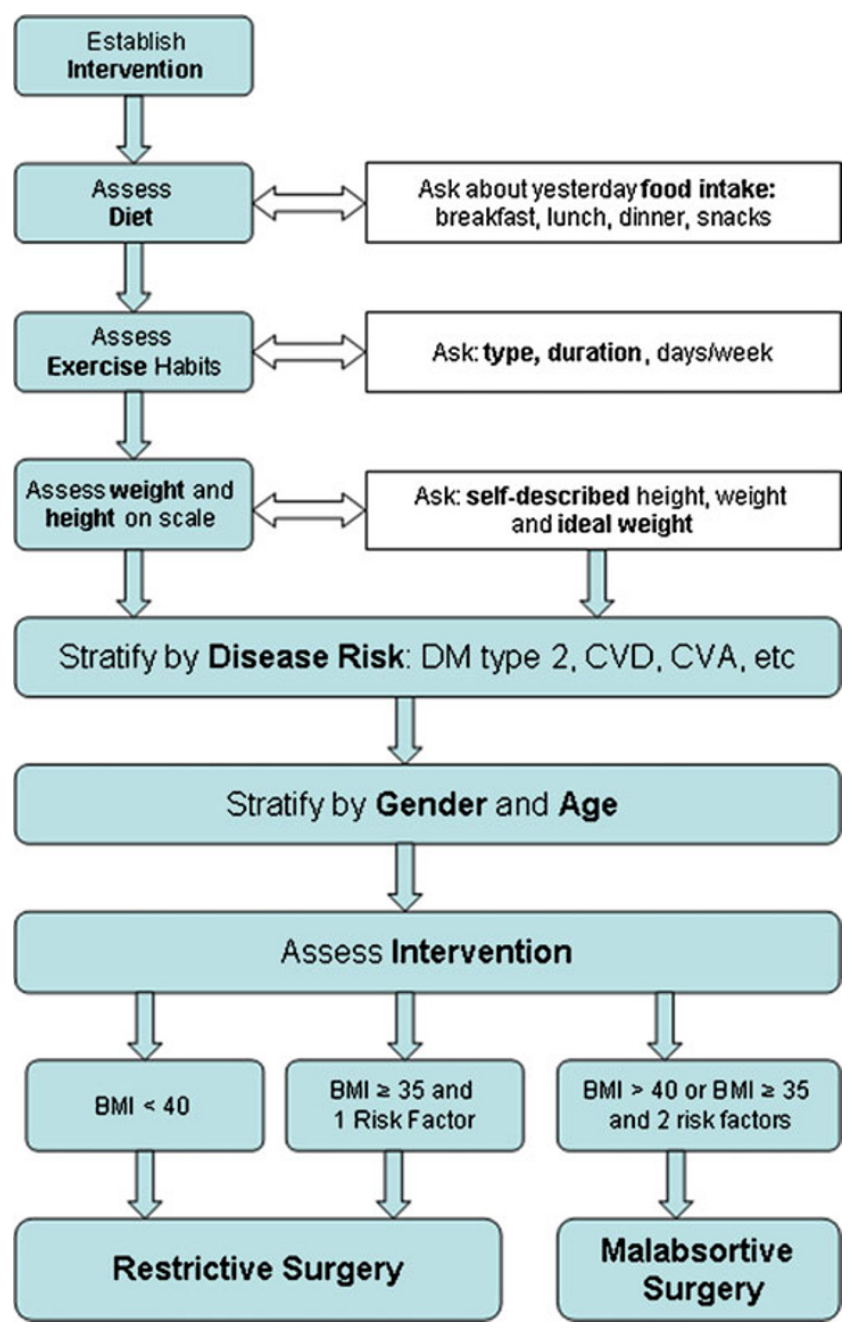

Fig. 1 Individualized approach for the bariatric patient: A flowchart example system that stratifies in a set of patients, based on a set of observations. The observations can include physical, biochemical, histological, genetic, and gene-expression data, among other types of information. Adjustments can be made to account for the possibility that observations of several patients may begin at different points in the progression of their respective disease processes. $D M$ diabetes mellitus, $C V D$ cardiovascular disease, $C V A$ cerebrovascular accident 
vascular diseases [4]. On average, patients lose $14-25 \%$ weight after bariatric surgery [5]. Patients who underwent gastric bypass surgery showed a significant decline in allcause mortality as well as coronary artery disease, diabetes and cancer during 7.1 years follow up [6]. Patients with recently diagnosed type 2 diabetes showed greater weight loss after gastric banding compared to conventional therapy (life style advice) as well as a greater chance of remission of type 2 diabetes [7]. This effect of bariatric surgery on diabetes is probably due to a reduction in body fat mass and, in the case of gastric bypass surgery, changes in gut hormone production such as Glucagon-Like Peptide-1 (GLP-1), Gastric Inhibitory Polypeptide (GIP) and grehlin [8]. GLP-1 receptor agonists induce adiponectin expression while reducing expression of IL-6 and MCP-1 in 3T3-L1 adipocytes through the protein kinase A pathway [9]. Finally, although only limited data is present, plasma GIP levels may important as seen from in vitro studies showing a GIP induced reduction in insulin resistance in 3T3-L1 adipocytes through activation of Akt [10]. Although only limited data is available, the effects of GLP-1 and GIP on adipocytes may be part of the weight loss independent effects of gastric bypass surgery on adipose tissue function. Other beneficial effects of gastric bypass surgery in comparison to gastric banding may include a smaller fat mass to fat-free mass ratio with similar weight loss [11]. Adiponectin levels have been shown to increase after bariatric surgery in several small scale studies mainly because of an increase in high molecular weight adiponectin [12]. After bariatric surgery, plasma concentrations of Macrophage Inhibitory Factor (MIF), Plasminogen Activator Inhibitor-1 (PAI-1), Retinol Binding Protein-4 (RBP-4), Monocyte Chemotactic Protein-1 (MCP-1) and interleukin-18 (IL-18) are decreased, indicating positive effects on adipose tissue function [13].

Dual-energy X-ray absorptiometry (DEXA) has been widely used in not only the diagnosis of osteoporosis but also the analysis of body composition such as the amount of bone mineral, adipose tissue, and other soft tissue. With this technique we can determine the exact amount of adipose tissue loss following bariatric surgery (Fig. 2).

\section{Bariatric medicine team}

A technically proficient team, preferably accredited by a national certifying organization, provides the necessary guarantees that patients will receive adequate instructions before, during and after surgery through the collaboration of a multidisciplinary team that allows for a greater assurance of long-term success and helps monitor weight regain.
Surgery

Bariatric surgery appears efficacious compared to standard care in reducing obesity. Weight losses are greatest with diversionary procedures, intermediate with diversionary/ restrictive procedures, and lowest with those that are purely restrictive. Compared with Roux-en-Y gastric bypass, adjustable gastric banding has lower weight loss efficacy, but also leads to fewer serious adverse effects. According to a recent survey, over $90 \%$ of bariatric procedures are performed by laparoscopic approach [14]. The most commonly performed procedures are Roux-en-Y gastric bypass (open and laparoscopic), followed by laparoscopic adjustable gastric banding, and sleeve gastrectomy. Definite geographic trends are observable in the specific bariatric procedures being performed. A number of studies have already demonstrated the efficacy of bariatric surgery for the treatment of obesity and its co-morbidities, although there are still only a handful of prospective, controlled studies with a high level of evidence. Considering the results derived from a large-scale, prospective, multicenter studies and a systematic review [15], it can be reasonably said that bariatric surgery is a safe and feasible intervention for the treatment of life-threatening morbid obesity under controlled conditions [16]. Since 2004, the American Society of Bariatric and Metabolic Surgery administers the bariatric surgery centers of excellence accredited worldwide. There are currently 441 centers and 758 surgeons accredited in the US and Canada. In Europe, accreditation of bariatric surgery centers of excellence is governed by the European Accreditation Council for Bariatric Surgery, a member of the IFSO-European Chapter (International Federation for the Surgery of Obesity and Metabolic Disorders-European Chapter). Fifty-four European centers are currently accredited by this institution.

\section{Nutritionist}

Postoperatively, patients experience nutritional, metabolic, and hormonal changes that have important clinical implications [17]. While purely restrictive procedures do not directly lead to deficiencies attributable to malabsoption, stomal stenosis resulting in markedly reduced food intake can lead to caloric, protein and other nutritional deficits. Thus patients should be monitored regularly following surgery. Menstruating women are vulnerable to developing iron deficiency anemia, and micronutrient deficiencies such as vitamin B12, folate and zinc deficiencies are common, especially following malabsorptive procedures. Bone metabolism is greatly affected, in part due to vitamin D deficiency, decreased calcium absorption, and secondary hyperparathyroidism. To prevent deficiencies oral multivitamin preparations should be given to patients undergoing 
Fig. 2 The two classes of surgical procedures most commonly used to produce weight loss are: a) gastric restriction (adjustable gastric binding) and b) malabsorbtion (Roux-en-Y gastric bypass and bilopancreatic diversion). Among variety of biomarkers used to monitor weight loss most clinically proven are: serum adiponectin and whole body DEXA scan. $A G B$ Adjustable Gastric Binding, RYGB Roux-en-Y Gastric Bypass, $B D P$ Bilopancreatic Diversion
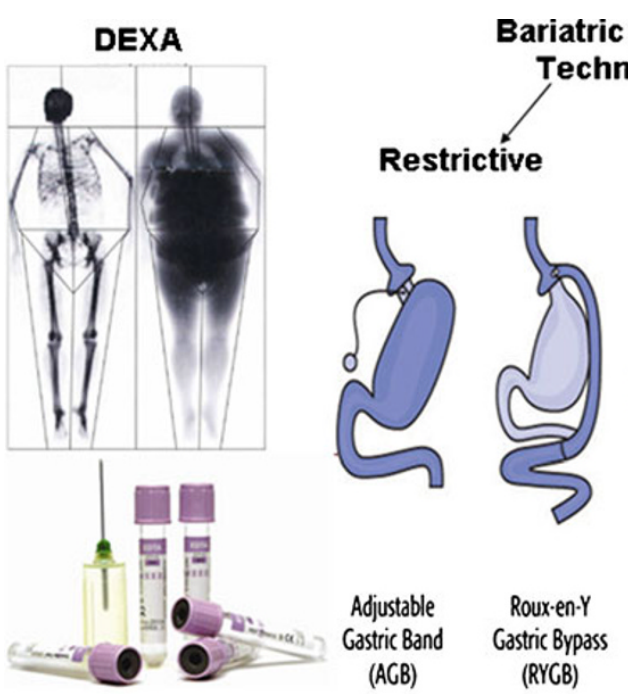

Biomarkers
DEXA
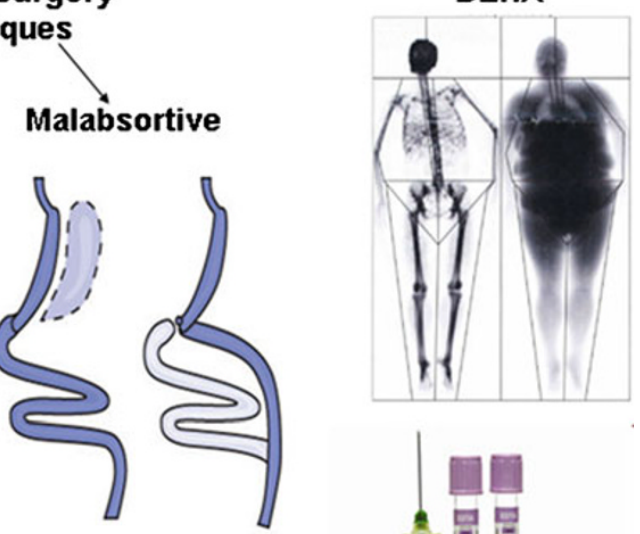

Vertical Sleeve (USG)

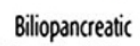
Diversion With a Duodenal Switch (BPD.DS)

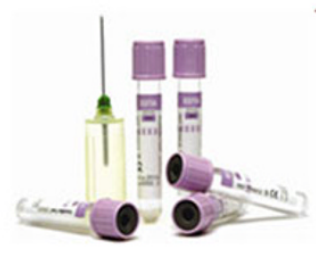

Biomarkers

\section{Assess DEXA and Biomarkers at Baseline and each $6^{\text {th }}$ month}

bariatric surgery [18]. Women should be placed on calcium supplement. Patients should be encouraged to consume 60 $120 \mathrm{~g}$ of protein daily to maintain lean body mass during weight loss and long-term maintenance [19]. A change in dietary orientation by selecting different protein sources (meat, fish, eggs, legumes with cereals, cheese, tofu...) combined with powder protein supplements may allow for achieving the expected protein intake.

\section{Endocrinologist}

Diabetes improves acutely in malabsorptive procedures and in sequence with weight loss in restrictive procedures. Bariatric surgery can significantly improve glycaemic control in severely obese patients with Type 2 diabetes. It is an effective, safe and cost-effective therapy for obese Type 2 diabetes. Panelists recommend that postoperative glycemic control should consist of achievement of preprandial blood glucose levels less than $110 \mathrm{mg} / \mathrm{dL}$ and postprandial blood glucose levels no greater than $180 \mathrm{mg} /$ $\mathrm{dL}$, in order to achieve glycated hemoglobin (HbAlc) values of $7 \%$ or less [20]. Adjusted with the recent IDF (International Diabetes Federation) recommendations national guidelines for bariatric surgery need to be developed for people with Type 2 diabetes and a BMI of $35 \mathrm{~kg} / \mathrm{m}$ (2) or more [21]. Polycystic ovarian syndrome improves in nearly all women with this condition who undergo bariatric surgery. Testosterone levels in men also improve after surgery. Consideration of these nutritional, metabolic, and hormonal changes allows for optimal medical management following bariatric surgery. Physical activity is a cornerstone in the medical management of obesity and could be important for weight loss following bariatric surgery. A literature search identified 20 publications (19 studies) reporting physical activity data in relation to bariatric surgery. Observational evidence of self-reported physical activity suggests that physical activity increases after bariatric surgery and that physical activity is associated with surgically induced weight loss [22].

\section{Cardiologist}

Weight loss by means of caloric restriction or surgery results in favorable hemodynamic changes referred to as "reverse remodeling". Regression of left ventricular (LV) mass and chamber size has been shown universally. However, some studies have failed to reveal improvement in diastolic function possibly because of confounders such as nutritional deficiency that may occur after weight loss surgery. Some evidence seems to suggest that the greatest regression of LV mass and LV hypertrophy may occur when weight loss is combined with beta-adrenergic blocker therapy (in those who have an indication for the drug) when compared with other antihypertensive drugs versus weight loss alone [23].

\section{Psychologist}

Although obesity is considered to be a medical condition, psychosocial variables influence its development, course, and treatment. To date, obesity research focuses heavily on the associated physical problems and the identification of effective treatments. While an array of behavioral, pharmacological and surgical treatments has been developed, their 
ability to demonstrate long-term weight losses is not convincing. Thus, there has been a shift towards the identification of psychological and social factors that may assist in the prediction of successful treatments, most notably in the surgical treatment of obesity. As a result, less emphasis has been placed on post-treatment psychosocial influences. As the treatment of obesity becomes increasing multidimensional, the need for attention to psychosocial factors and the involvement of mental health providers increases. For the allied or mental health provider, a comprehensive understanding of obesity's physical, psychological and social basis is vital to ensure proper assessment or treatment, as it is warranted. More information is needed regarding factors that interfere with successful outcomes and mechanisms of optimal follow-up for bariatric surgery patients to prevent and detect post-operative medical, psychological and social difficulties [24].

\section{Conclusion and outlook}

According to a report (Shedding the pounds "Obesity management, NICE guidance and bariatric surgery in England") released by the UK Office of Health Economics, an independent research body, a big increase in bariatric surgery among people who meet the current criteria would pay for itself in 1 year, by enabling many of them to return to paid employment [25]. According to this report, around one million people in England currently meet the criteria for bariatric surgery recommended by the National Institute for Health and Clinical Excellence, of whom only 5\% to $10 \%$ may actually be eligible for surgery. If just $5 \%$ of eligible patients were to receive bariatric surgery the net gain to the UK economy within 3 years could be $£ 382 \mathrm{~m}$. Critics of the report point out that it was commissioned by the Royal College of Surgeons of England together with the National Obesity Forum and Allergan and Covidien, companies that make products for obesity surgery. Critics also note that this money would be better spent on prevention efforts (though they don't present any data showing that such efforts will actually prevent current costs of people who are already obese). John Ashton, the chairman of the UK Public Health Association, is quoted in the British Medical Journal as raising the issue of opportunity cost of spending money on surgery that is then not available for other health services and conditions that are not preventable. This is a moot argument as, irrespective of whether or not obesity is preventable, not spending the money on surgery for those who already have the problem, will just mean spending more money on managing their many complications, e.g. type 2 diabetes mellitus.

In addition, the International Diabetes Federation (IDF) published in April 2011 Executive Summary pointing out that "Bariatric surgery is an appropriate treatment for people with type 2 diabetes and obesity not achieving recommended treatment targets with medical therapies, especially when there are other major co-morbidities" [21].

The Endocrine Society recently released a clinical practice guideline that highlights the need for endocrine and nutritional management of patients after bariatric surgery, including those with diabetes [26]. Given the large number of people undergoing bariatric surgery, it is important for endocrinologists and primary care physicians to be knowledgeable about the postoperative care of these patients. The patients have a risk for weight regain if they do not participate in ongoing dietary and behavioral care in the long term. One of the key recommendations in the guideline is the need for a multidisciplinary team to oversee the ongoing dietary needs of patients after surgery. This should not be done by the surgeons alone. The team should include psychologists, nutritionists, endocrinologists, etc. to evaluate patients, as many patients present with different problems.

Emerging bariatric techniques are: implantable gastric stimulator and VBLOC therapy with surgically implanted device that delivers high-frequency, low energy electrical signals. It is thought that weight loss is mediated by interruption of hunger signals that travel via the vagus nerve to the brain, though the exact mechanism of action has yet to be elucidated.

With the growing interest in bariatric surgery not only are primary care practitioners face with an increasing number of requests for referral to surgery but they are called upon to look after an ever increasing number of patients who have undergone bariatric surgery [27]. In this article we provide a succinct overview of how multidisciplinary team should prepare the selection criteria for surgery, types of surgery, risks and benefits, long-term complications, as well as monitoring and management issue relevant to the care of these complex patients. While it is impossible to summarize the vast and rapidly expanding literature on bariatric surgery in a short article, we do hope that this paper will provide a useful resource to anyone seeking a quick state-of-the-art overview of bariatric medicine.

\section{References}

1. Ogden CL, Yanovski SZ, Carroll MD, Flegal KM. The epidemiology of obesity. Gastroenterology. 2007;132:2087-102.

2. de la Cruz-Muñoz N, Messiah SE, Arheart KL, Lopez-Mitnik G, Lipshultz SE, Livingstone A. Bariatric surgery significantly decreases the prevalence of type 2 diabetes mellitus and prediabetes among morbidly obese multiethnic adults: long-term results. J Am Coll Surg. 2011;212(4):505-11. discussion 512-3.

3. Franco JV, Ruiz PA, Palermo M, Gagner M. A review of studies comparing three laparoscopic procedures in bariatric surgery: 
sleeve gastrectomy, Roux-en-Y gastric bypass and adjustable gastric banding. Obes Surg. 2011. doi:10.1007/s11695-011-0390-5.

4. Demaria EJ. Bariatric surgery for morbid obesity. N Engl J Med. 2007;356:2176-83.

5. Sjostrom L, Narbro K, Sjostrom CD, Karason K, Larsson B, Wedel H, et al. Effects of bariatric surgery on mortality in Swedish obese subjects. N Engl J Med. 2007;357:741-52.

6. Adams TD, Gress RE, Smith SC, Halverson RC, Simper SC, Rosamond WD, et al. Long-term mortality after gastric bypass surgery. N Engl J Med. 2007;357:753-61.

7. Dixon JB, O’Brien PE, Playfair J, Chapman L, Schachter LM, Skinner $\mathrm{S}$, et al. Adjustable gastric banding and conventional therapy for type 2 diabetes: a randomized controlled trial. JAMA. 2008;299:316-23.

8. Vetter ML, Cardillo S, Rickels MR, Iqbal N. Narrative review: effect of bariatric surgery on type 2 diabetes mellitus. Ann Intern Med. 2009;150:94-103.

9. Kim Chung IT, Hosaka T, Yoshida M, Harada N, Sakaue H, Sakai $\mathrm{T}$, et al. Exendin-4, a GLP-1 receptor agonist, directly induces adiponectin expression through protein kinase A pathway and prevents inflammatory adipokine expression. Biochem Biophys Res Commun. 2009;390:613-8.

10. Song DH, Getty-Kaushik L, Tseng E, Simon J, Corkey BE, Wolfe MM. Glucose-dependent insulinotropic polypeptide enhances adipocyte development and glucose uptake in part through Akt activation. Gastroenterology. 2007;133:1796-805.

11. Lubrano C, Mariani S, Badiali M, Cuzzolaro M, Barbaro G, Migliaccio S, et al. Metabolic or bariatric surgery? Long-term effects of malabsorptive vs restrictive bariatric techniques on body composition and cardiometabolic risk factors. Int J Obes (Lond). 2010;34:1404-14.

12. Diker D, Vishne T, Maayan R, Weizman A, Vardi P, Dreznik Z, et al. Impact of gastric banding on plasma adiponectin levels. Obes Surg. 2006;16:1057-61.

13. Schernthaner GH, Kopp HP, Kriwanek S, Krzyzanowska K, Satler M, Koppensteiner R, et al. Effect of massive weight loss induced by bariatric surgery on serum levels of interleukin-18 and monocytechemoattractant-protein-1 in morbid obesity. Obes Surg. 2006;16:709-15.

14. Buchwald H, Oien DM. Metabolic/bariatric surgery worldwide 2008. Obes Surg. 2009;19:1605-11.
15. Padwal R, Klarenbach S, Wiebe N, Birch D, Karmali S, Manns B, et al. Bariatric surgery: a systematic review and network meta-analysis of randomized trials. Obes Rev. 2011. doi:10.1111/j.1467-789X. 2011.00866.x.

16. Kelles SM, Barreto SM, Guerra HL. Mortality and hospital stay after bariatric surgery in 2,167 patients: influence of the surgeon expertise. Obes Surg. 2009;19:1228-35.

17. Shankar P, Boylan M, Sriram K. Micronutrient deficiencies after bariatric surgery. Nutrition. 2010;26(11-12):1031-7. Epub 2010 Apr 3.

18. Rubio MA, Moreno C. Nutritional implications of bariatric surgery on the gastrointestinal tract. Nutr Hosp. 2007;22 Suppl 2:124-34.

19. Andreu A, Moizé V, Rodríguez L, Flores L, Vidal J. Protein intake, body composition, and protein status following bariatric surgery. Obes Surg. 2010;20:1509-15. 20.

20. American Diabetes Association. Standards of medical care in diabetes-2011. Diabetes Care. 2011;34 suppl 1:S11-61.

21. Dixon JB, Zimmet P, Alberti KG, Rubino F, on behalf of the International Diabetes Federation Taskforce on Epidemiology and Prevention. Bariatric surgery: an IDF statement for obese Type 2 diabetes. Diabet Med. 2011. doi:10.1111/j.1464-5491. 2011.03306.x.

22. Jacobi D, Ciangura C, Couet C, Oppert JM. Physical activity and weight loss following bariatric surgery. Obes Rev. 2011;12 (5):366-77.

23. Poirier P, Cornier MA, Mazzone T, Stiles S, Cummings S, Klein S, et al. Bariatric surgery and cardiovascular risk factors: a scientific statement from the American Heart Association. Circulation. 2011;123(15):1683-701.

24. Pataky Z, Carrard I, Golay A. Psychological factors and weight loss in bariatric surgery. Curr Opin Gastroenterol. 2011;27(2):16773.

25. Obesity management, NICE guidance and bariatric surgery in England. UK Office of Health Economics 2010. www.ohe.org/lib/ liDownload/692/OHE_Spreads.pdf

26. Heber D, Greenway FL, Kaplan LM, Livingston E, Salvador J, Still $\mathrm{C}$, et al. Endocrine and nutritional management of the postbariatric surgery patient: an Endocrine Society Clinical Practice Guideline. J Clin Endocrinol Metab. 2010;95(11):4823-43.

27. Brown W, Korin A, Burton P, O'Brien PE. Laparoscopic adjustable gastric banding. Aust Fam Physician. 2009;38(12):972-6. 